\title{
Testing a simple criterion for the critical temperature
}

\author{
Ivo Souza, ${ }^{*}$ Carlos Lobo, ${ }^{*}$ and João Seixas \\ Departamento de Física, Instituto Superior Técnico, Av. Rovisco Pais 1, 1096 Lisboa codex, Portugal
}

(Received 25 March 1996; revised manuscript received 31 October 1996)

\begin{abstract}
We investigate the applicability of a simple criterion proposed by Wosiek [Phys. Rev. B 49, 15023 (1994)] for locating analytically critical temperatures; we apply it to Ising-like spin models with anisotropic interactions, more than one type of interaction between the spins, and multiple-spin interactions. The predicted critical temperatures only agree with the known exact results or with Monte Carlo simulations for very particular, highly symmetrical values of the coupling constants. [S0163-1829(97)02809-9]
\end{abstract}

\section{INTRODUCTION}

Wosiek $^{1}$ recently proposed the following ansatz for locating the critical temperature $T_{c}$ of a lattice spin system: let us define, as usual, $\beta \equiv 1 / K_{B} T$, let $T$ be a transfer matrix of a $d$-dimensional system $(d>1)$ having a finite degeneracy in the ground state-this excludes models in which the spins can take continuous values - and let $L$ be its linear size. Defining the "characteristic function",

$$
\rho(\beta)=\lim _{L \rightarrow \infty}\left[\frac{(\operatorname{Tr} T)^{2}}{\operatorname{Tr} T^{2}}\right]^{1 / L^{(d-1)}}
$$

the maximum of $\rho$ occurs at the order-disorder phase transition point ("maximum principle"): $\beta_{\max }=\beta_{c}$.

The maximum principle can be reformulated in an equivalent way: ${ }^{1}$ let us consider the normalized second moment at finite $L, r_{L}(\beta)=(\operatorname{Tr} T)^{2} / \operatorname{Tr} T^{2}=\left(Z_{1}\right)^{2} / Z_{2}$. It is easy to see ${ }^{1}$ that $Z_{1}$ and $Z_{2}$ are the partition functions of a $(d-1)$ dimensional system, and of two coupled $(d-1)$-dimensional systems, respectively. What systems are these depends on the choice of $T$, as will become clear latter. Differentiation of the logarithm of $r_{L}(\beta)$ gives as the condition for the maximum $u_{2}\left(\beta_{\max }\right)=u_{1}\left(\beta_{\max }\right)$, where $u_{1}$ and $u_{2}$ denote the energy density of the $(d-1)$-dimensional system and of the two coupled $(d-1)$-dimensional systems, respectively.

In this paper we will test the maximum principle in some simple models; the choice of models was made in order to test the criterion in distinct situations: in Sec. II we study an anisotropic self-dual system in two dimensions; Wosiek argued that his method is exact for self-dual systems with $d=2$, but he considered isotropic models only. In Sec. III we look at a system with more than one type of interaction between the spins, one of them involving more than two spins. In Sec. IV we study a system in a triangular lattice with multiple-spin interactions. In Sec. V we analyze the AshkinTeller model, which displays two critical points for certain values of the parameters. In all of these models, for a certain range of the coupling parameters at least, the location of the critical point is a consequence of symmetries like self-duality. ${ }^{2}$ Finally, in Sec. VI we briefly report a numerical study of the two-layer Ising model, for which Wosiek had predicted the critical temperature $\beta_{c} \cong 0.2656$. ${ }^{1}$ As Wosiek points out, this is an important example because not only is the system not self-dual, but apparently it does not have any more general symmetry, and thus provides a test for the possibility of the maximum rule being a consequence of such symmetries.

\section{TWO-DIMENSIONAL ANISOTROPIC ISING MODEL IN A SQUARE LATTICE}

As is well known, the critical temperature of this model is obtained from self-duality, ${ }^{2}$ and is given by $\sinh \left(2 \beta_{c} J_{1}\right) \sinh \left(2 \beta_{c} J_{2}\right)=1$. We will analyze this system using three different methods; the first and the third differ in the choice of the transfer matrix, while the second is a generalization of the maximum principle. Results from the three procedures are plotted together in Fig. 1.

Method 1. For a lattice with linear size $L$, the partition function is

$$
Z_{L}=\Sigma_{s} \exp \left(\beta \sum_{i=1}^{L} \sum_{j=1}^{L} J_{1} s_{i j} s_{i+1 j}+J_{2} s_{i j} s_{i j+1}\right)
$$

where periodic boundary conditions in both directions are implied and $J_{1}\left(J_{2}\right)$ is the horizontal (vertical) coupling constant. Choosing $T$ to propagate the rows of the lattice, it is straightforward to calculate the matrix elements $\left\langle\phi|T| \phi^{\prime}\right\rangle$, where $\phi$ and $\phi^{\prime}$ are vectors containing rows of spins. $\operatorname{Tr} T$ is

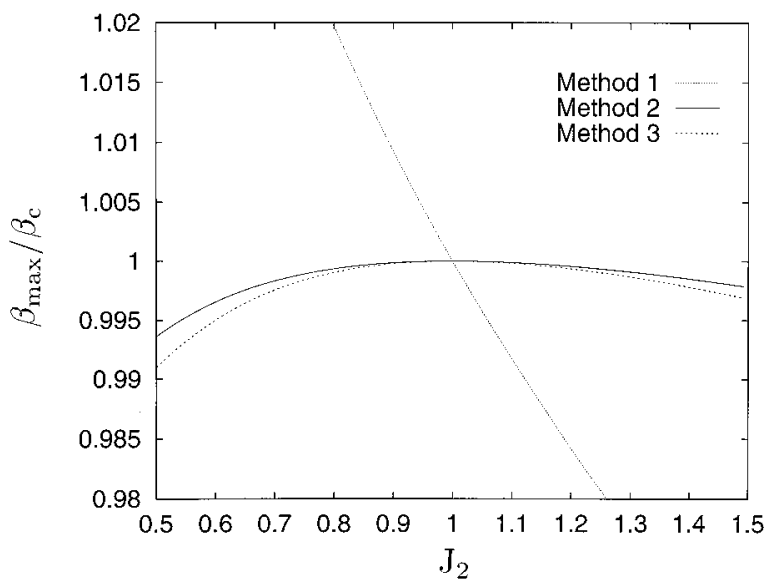

FIG. 1. $\beta_{\max } / \beta_{c}$ as a function of $J_{2}$, with $J_{1}=1$, for the twodimensional anisotropic Ising model, using the three methods described in Sec. II. 
the partition function $Z_{1}$ of a horizontal one-dimensional spin chain with periodic boundary conditions in both directions (each spin couples with itself along the vertical direction); hence $Z_{1}=\operatorname{Tr}\left(T_{1}\right)^{L}$. Similarly, $\operatorname{Tr} T^{2}$ is the partition function $Z_{2}$ of two coupled horizontal chains of spins, again with horizontal and vertical periodic boundary conditions: $Z_{2}=\operatorname{Tr}\left(T_{2}\right)^{L}$. The matrices $T_{1}$ and $T_{2}$ are given by

$$
\begin{gathered}
\left\langle s\left|T_{1}\right| s^{\prime}\right\rangle=\exp \left[\beta\left(J_{1}+J_{2} s s^{\prime}\right)\right], \\
\left\langle s_{1} s_{2}\left|T_{2}\right| s_{1}^{\prime} s_{2}^{\prime}\right\rangle=\exp \left\{\beta\left[2 J_{1} s_{1} s_{2}+J_{2}\left(s_{1} s_{1}^{\prime}+s_{2} s_{2}^{\prime}\right)\right]\right\} .
\end{gathered}
$$

We will only need the largest eigenvalues of $T_{1}$ and $T_{2}$ because they are dominant in taking the limit in Eq. (1): $\rho(\beta)=t_{1 \max }^{2} / t_{2 \max }$. With the help of MATHEMATICA, we get

$$
\begin{gathered}
t_{1 \max }=x^{\left(J_{1}+J_{2}\right)}+x^{\left(J_{1}-J_{2}\right)}, \\
t_{2 \max }=\frac{1}{2}\left[x^{2 J_{2}-2 J_{1}}+x^{-2 J_{1}-2 J_{2}}+x^{2 J_{1}+2 J_{2}}+x^{2 J_{1}-2 J_{2}}\right] \\
+\frac{1}{2}\left[\left(x^{2 J_{1}+2 J_{2}}+x^{2 J_{1}-2 J_{2}}\right)^{2}+x^{-4 J_{1}+4 J_{2}}+x^{-4 J_{1}-4 J_{2}}\right. \\
\left.-2\left(x^{4 J_{2}}+x^{-4 J_{2}}\right)+2 x^{-4 J_{1}}+12\right]^{1 / 2},
\end{gathered}
$$

where $x \equiv e^{\beta}$ (this definition will be used henceforth).

From Fig. 1 we see that $\beta_{\max }=\beta_{c}$ only when $J_{1}=J_{2}$. Whereas the exact solution is obviously symmetrical in $J_{1}$ and $J_{2}$, this is not the case with Eqs. (4), the reason being that the one-dimensional spin chain and the two coupled onedimensional spin chains we used were horizontal, and so $J_{1}$ and $J_{2}$ played distinct roles. As a consequence, the conditions $u_{1}^{h}\left(\beta_{\max }\right)=u_{2}^{h}\left(\beta_{\max }\right)$ and $u_{1}^{\nu}\left(\beta_{\max }\right)=u_{2}^{\nu}\left(\beta_{\max }\right)$ yield different values for $\beta_{\max }$ (the indices $h$ and $v$ label the horizontal and vertical spin chains). We can recover that symmetry by considering both horizontal and vertical spin chains simultaneously; one possibility is to use the condition $u_{2}^{h}\left(\beta_{\max }\right)+u_{2}^{\nu}\left(\beta_{\max }\right)=u_{1}^{h}\left(\beta_{\max }\right)+u_{1}^{\nu}\left(\beta_{\max }\right)$ instead (Method 2$)$. This amounts to replacing the partition functions $Z_{1,2}$ by the geometric mean of $Z_{1,2}^{h}$ and $Z_{1,2}^{\nu}$ in the expression for the second-order moment of the transfer matrix, giving

$$
\rho(\beta)=\frac{t_{1 \max }^{h} t_{1 \max }^{\nu}}{\sqrt{t_{2 \max }^{h} t_{2 \max }^{\nu}}}
$$

Once again the predicted critical temperatures match the exact values only in the isotropic case, but otherwise the deviations are much smaller than those corresponding to the first method.

Method 3. If we choose a transfer matrix that propagates diagonal chains of spins (considering now a lattice with linear dimensions $n$ along the diagonal of the original lattice), we obtain in the end a different expression for $\rho(\beta)$, which is symmetrical in $J_{1}$ and $J_{2}$, as required. Following Baxter, ${ }^{2}$ we label the transfer matrix $V W$, where $V$ and $W$ are the matrices

$$
\left\langle\phi^{A}|V| \phi^{B}\right\rangle=\exp \left(\beta \sum_{j=1}^{n} J_{1} s_{j+1}^{A} s_{j}^{B}+J_{2} s_{j}^{A} s_{j}^{B}\right)
$$

$$
\left\langle\phi^{B}|W| \phi^{A}\right\rangle=\exp \left(\beta \sum_{j=1}^{n} J_{1} s_{j}^{B} s_{j}^{A}+J_{2} s_{j}^{B} s_{j+1}^{A}\right),
$$

and the partition function is $Z_{n}=\operatorname{Tr}(V W)^{n / 2}$. Using Eq. (5) we obtain

$$
\operatorname{Tr}(V W)=\sum_{\phi^{A}} \sum_{\phi^{B}} \prod_{j=1}^{n} \exp \left[\beta\left(J_{1}+J_{2}\right) s_{j}^{B}\left(s_{j}^{A}+s_{j+1}^{A}\right)\right]
$$

Equation (6) is the partition function $Z_{1}$ of a diagonal spin chain with periodic boundary conditions. Again, we define $\operatorname{Tr} T^{2}=Z_{2}$. The corresponding transfer matrices are

$$
\begin{gathered}
\left\langle s_{1} s_{2}\left|T_{1}\right| s_{1}^{\prime} s_{2}^{\prime}\right\rangle=\exp \left[\beta\left(J_{1}+J_{2}\right) s_{2}\left(s_{1}+s_{1}^{\prime}\right)\right], \\
\left\langle s_{1} s_{2} s_{3} s_{4}\left|T_{2}\right| s_{1}^{\prime} s_{2}^{\prime} s_{3}^{\prime} s_{4}^{\prime}\right\rangle \\
=\exp \left\{\beta \left[J_{1}\left(s_{1}^{\prime} s_{3}+s_{2}^{\prime} s_{4}+s_{2} s_{3}+s_{1} s_{4}\right)\right.\right. \\
\left.\left.+J_{2}\left(s_{1}^{\prime} s_{4}+s_{2}^{\prime} s_{3}+s_{1} s_{3}+s_{2} s_{4}\right)\right]\right\}
\end{gathered}
$$

with largest eigenvalues

$$
\begin{aligned}
& t_{1 \max }=x^{-2\left(J_{1}+J_{2}\right)}+x^{2\left(J_{1}+J_{2}\right)}+2, \\
t_{2 \max }= & \frac{1}{2} x^{-8\left(J_{1}+J_{2}\right)}\left[q_{1}(x)+x^{4\left(J_{1}+J_{2}\right)}\left(1+x^{4 J_{1}}\right)\right. \\
& \left.\times\left(1+x^{4 J_{2}}\right) \sqrt{q_{2}(x)}\right]
\end{aligned}
$$

where

$$
\begin{aligned}
q_{1}(x)= & x^{4\left(J_{1}+J_{2}\right)}+x^{12 J_{1}+4 J_{2}}+12 x^{8\left(J_{1}+J_{2}\right)}+x^{4 J_{1}+12 J_{2}} \\
& +x^{12\left(J_{1}+J_{2}\right)}, \\
q_{2}(x)= & 1-2\left(x^{4 J_{1}}+x^{4 J_{2}}\right)+x^{8 J_{1}}+x^{8 J_{2}}+20 x^{4\left(J_{1}+J_{2}\right)} \\
& -2 x^{8 J_{1}+4 J_{2}}-2 x^{4 J_{1}+8 J_{2}}+x^{8\left(J_{1}+J_{2}\right)} .
\end{aligned}
$$

$T_{2}$ was diagonalized using the property $\left[T_{2}, U\right]$ $=0$, where $U=\left(\sigma_{x} \otimes \sigma_{x}\right) \otimes\left(\sigma_{x} \otimes \sigma_{x}\right)=\delta\left(s_{1},-s_{1}^{\prime}\right) \delta\left(s_{2}\right.$, $\left.-s_{2}^{\prime}\right) \delta\left(s_{3},-s_{3}^{\prime}\right) \delta\left(s_{4},-s_{4}^{\prime}\right)$ is the matrix associated with the inversion of the spins in a $16 \times 16$ spin matrix ( $\sigma_{x}$ is the Pauli matrix). We see that $\rho(\beta)$ is now symmetrical in $J_{1}$ and $J_{2}$, as expected. Once again the results are correct for the isotropic case only, and for $J_{1} \neq J_{2}$ the previous method gives slightly better values.

\section{ISING-TYPE MODEL IN A SQUARE LATTICE WITH NEAREST-NEIGHBOR, NEXT-NEAREST-NEIGHBOR, AND FOUR-SPIN (SQUARE) INTERACTIONS}

Choosing the transfer matrix to propagate vertically the rows of the lattice, we find

$$
\begin{aligned}
\left\langle s\left|T_{1}\right| s^{\prime}\right\rangle= & \exp \left\{\beta\left[J_{1}+\left(J_{1}+2 J_{2}\right) s s^{\prime}+J_{3}\right]\right\}, \\
\left\langle s_{1} s_{2}\left|T_{2}\right| s_{1}^{\prime} s_{2}^{\prime}\right\rangle= & \exp \left\{\beta \left[J_{1}\left(s_{1} s_{2}+s_{1} s_{1}^{\prime}+s_{2} s_{2}^{\prime}+s_{1}^{\prime} s_{2}^{\prime}\right)\right.\right. \\
& \left.\left.+2 J_{2}\left(s_{1} s_{2}^{\prime}+s_{1}^{\prime} s_{2}\right)+2 J_{3}\left(s_{1} s_{1}^{\prime} s_{2} s_{2}^{\prime}\right)\right]\right\},
\end{aligned}
$$


where $J_{1}, J_{2}$, and $J_{3}$ are the coupling constants for nearestneighbor, next-nearest-neighbor, and four-spin interactions, respectively. The largest eigenvalues are

$$
\begin{aligned}
& t_{1 \max }=x^{2 J_{1}+2 J_{2}+J_{3}}+x^{-2 J_{2}+J_{3}}, \\
t_{2 \max }= & \frac{1}{2}\left[x^{4 J_{1}+4 J_{2}+2 J_{3}}+2 x^{-4 J_{2}+2 J_{3}}\right. \\
& +x^{-4 J_{1}+4 J_{2}+2 J_{3}}+\left(x^{8 J_{1}+8 J_{2}+4 J_{3}}\right. \\
& \left.\left.+16 x^{-4 J_{3}}-2 x^{8 J_{2}+4 J_{3}}+x^{-8 J_{1}+8 J_{2}+4 J_{3}}\right)^{1 / 2}\right] .
\end{aligned}
$$

$T_{2}$ was diagonalized by noting that it remains unchanged when the spin labels 1 and 2 are exchanged, and so it commutes with the $4 \times 4$ matrix with elements $\delta\left(s_{1}, s_{2}^{\prime}\right) \delta\left(s_{2}, s_{1}^{\prime}\right)$.

The case $J_{1}=J_{2}=0$ is ruled out because it has infinite degeneracy in the ground state. For $J_{1}=J_{3}=0$, the lattice factors into two independent nearest-neighbor isotropic square Ising lattices, and thus we would expect to recover the well-known result $\beta_{c} J_{2}=0.4407 \ldots$. Instead we get $\rho(\beta)=1$, and thus no prediction for $\beta_{c}$ can be exctracted from the maximum principle. The case $J_{1}=0$ corresponds to the isotropic zero-field eight-vertex model, for which the exact value for $\beta_{c}$ in the ferromagnetic regime can be derived using a generalization of the star-triangle relations, ${ }^{2}$ and is given by

$\exp \left[\beta_{c}\left(2 J_{2}+J_{3}\right)\right]=\exp \left[\beta_{c}\left(-2 J_{2}+J_{3}\right)\right]+2 \exp \left(-\beta_{c} J_{3}\right)$.

Only for $J_{2}=J_{3}$ does the maximum principle reproduce the correct result.

\section{TRIANGULAR THREE-SPIN MODEL}

The energy is given by $E=-\sum s_{i} s_{j} s_{k}$, where the sum is over all triangular faces of the triangular lattice. The transfer matrices

$$
\begin{gathered}
\left\langle s\left|T_{1}\right| s^{\prime}\right\rangle=\exp \left[\beta\left(s+s^{\prime}\right)\right] \\
\left\langle s_{1} s_{2}\left|T_{2}\right| s_{1}^{\prime} s_{2}^{\prime}\right\rangle=\exp \left\{\beta\left[s_{1} s_{2}\left(s_{1}^{\prime}+s_{2}^{\prime}\right)+s_{1}^{\prime} s_{2}^{\prime}\left(s_{1}+s_{2}\right)\right]\right\}
\end{gathered}
$$

have largest eigenvalues

$$
\begin{gathered}
t_{1 \max }=x^{2}+x^{-2}, \\
t_{2 \max }=\frac{1+2 x^{4}+x^{8}}{3 x^{4}}+\frac{1+4 x^{4}+6 x^{8}+4 x^{12}+x^{16}}{3 x^{4} \sqrt[3]{q_{1}(x)}}+\frac{\sqrt[3]{q_{1}(x)}}{3 x^{4}},
\end{gathered}
$$

with

$$
\begin{gathered}
q_{1}(x)=1-21 x^{4}+15 x^{8}+74 x^{12}+15 x^{16}-21 x^{20}+x^{24} \\
+3^{3 / 2} x^{2} \sqrt{q_{2}(x)}, \\
q_{2}(x)=-2+15 x^{4}-26 x^{8}-124 x^{12}+28 x^{16}+218 x^{20}+28 x^{24} \\
-124 x^{28}-26 x^{32}+15 x^{36}-2 x^{40} .
\end{gathered}
$$

The maximum of $\rho(\beta)$ is located at $\beta_{\max }=0.4407 \ldots$, which agrees with $\beta_{c}{ }^{2}$ We note that the critical temperature of this system can be obtained using a duality relation. ${ }^{2}$

If we allow the coupling constants for the up-pointing and down-pointing triangles to take distinct values, we arrive at a rather long expression for $\rho(\beta)$ which is symmetrical in the coupling constants, but fails to provide the right answer (we compared the values of $\beta_{\max }$ with the results from Monte Carlo simulations). We do not know if this more general model obeys a symmetry relation which determines its critical point.

\section{SQUARE-LATTICE ASHKIN-TELLER MODEL}

This model can be expressed in terms of Ising spins by associating with each site $i$ two spins, $s_{i}$ and $\sigma_{i}{ }^{2}$ The interaction energy for the edge $(i, j)$ is $\varepsilon(i, j)=-J s_{i} s_{j}$ $-J^{\prime} \sigma_{i} \sigma_{j}-J_{4} s_{i} \sigma_{i} s_{j} \sigma_{j}-J_{0}$ and the transfer matrices are

$$
\begin{aligned}
\left\langle s \sigma\left|T_{1}\right| s^{\prime} \sigma^{\prime}\right\rangle= & \exp \left\{\beta \left[J\left(1+s s^{\prime}\right)+J^{\prime}\left(1+\sigma \sigma^{\prime}\right)\right.\right. \\
& \left.\left.+J_{4}\left(1+s s^{\prime} \sigma \sigma^{\prime}\right)+J_{0}\right]\right\}, \\
\left\langle s_{1} \sigma_{1} s_{2} \sigma_{2}\left|T_{2}\right| s_{1}^{\prime} \sigma_{1}^{\prime} s_{2}^{\prime} \sigma_{2}^{\prime}\right\rangle & \\
= & \exp \left\{\beta \left[J\left(2 s_{1} s_{2}+s_{1} s_{1}^{\prime}+s_{2} s_{2}^{\prime}\right)\right.\right. \\
& +J^{\prime}\left(2 \sigma_{1} \sigma_{2}+\sigma_{1} \sigma_{1}^{\prime}+\sigma_{2} \sigma_{2}^{\prime}\right) \\
& \left.\left.+J_{4}\left(s_{1} \sigma_{1} s_{1}^{\prime} \sigma_{1}^{\prime}+s_{2} \sigma_{2} s_{2}^{\prime} \sigma_{2}^{\prime}+2 s_{1} \sigma_{1} s_{2} \sigma_{2}\right)+2 J_{0}\right]\right\} .
\end{aligned}
$$

The largest eigenvalue of $T_{1}$ is $t_{1 \max }=x^{J_{0}}\left[x^{2 J}+x^{2 J^{\prime}}+x^{2 J_{4}}\right.$ $\left.+x^{2\left(J+J^{\prime}+J_{4}\right)}\right]$. We did not manage to calculate $t_{2 \max }$ analytically, and thus studied $\rho(\beta)$ numerically. We proceeded as follows: $T_{2}$ is invariant under the inversion of all the spins (and so it commutes with the matrix $U$ defined in Sec. II); using this property, $T_{2}$ can be factored in two independent $8 \times 8$ submatrices. $T_{2}$ is also invariant under the exchange $s_{1} \leftrightarrow \sigma_{1}, s_{2} \leftrightarrow \sigma_{2}$ [and so it commutes with $V^{2}$, where $V$ $=\delta\left(s_{1}, s_{2}^{\prime}\right) \delta\left(s_{2}, s_{3}^{\prime}\right) \delta\left(s_{3}, s_{4}^{\prime}\right) \delta\left(s_{4}, s_{1}^{\prime}\right)$ is the matrix associated with the cyclic permutation of the indices]. Using these two symmetries, in the isotropic case, $J=J^{\prime}$, we were able to decompose $T_{2}$ into smaller matrices, with the largest eigenvalue belonging to a $6 \times 6$ submatrix. For a given value of $\beta$, we calculated numerically the largest eigenvalue, obtained a plot of $\rho(\beta)$, and located approximately its maximum by inspection. For the anisotropic case we did the same, but using the $8 \times 8$ submatrices mentioned above. In this latter case, it has been argued that the system always has two distinct critical temperatures, for any value of the coupling constants, ${ }^{2}$ and so we would expect to find two maximums of $\rho(\beta)$, but there was only one (in particular, for $J_{4}=0$, the system factors into two independent Ising lattices with coupling constants $J$ and $J^{\prime}$ ). In the anisotropic case the critical temperatures cannot be obtained from a duality relation, since such a symmetry implies the existence of a single critical temperature. When $J=J^{\prime}$, the criticality condition is obtained from a duality relation, ${ }^{2}$ and is given by $\exp \left(-2 \beta_{c} J_{4}\right)=\sinh \left(2 \beta_{c} J\right)$, with $J_{4}<J$.

We compare in Table I the exact values of $e^{\beta_{c}}$ with numerical values for $e^{\beta_{\max }}$, finding that in the limiting cases 
TABLE I. Comparison between the exact values of $e^{\beta_{c}}$ and those predicted by the maximum principle, $e^{\beta_{\max }}$, for the isotropic Ashkin-Teller model with $J=1$.

\begin{tabular}{ccc}
\hline \hline$J_{4}$ & \multicolumn{1}{c}{$e^{\beta_{c}}$} & \multicolumn{1}{c}{$e^{\beta_{\max }}$} \\
\hline 1 & $1.31607401 \ldots$ & ] $1.31607395,1.31607405[$ \\
0.9 & $1.32872007 \ldots$ & ] $1.32869336,1.32869372[$ \\
0.8 & $1.3427154 \ldots$ & ] $1.3425883,1.34258835[$ \\
0.7 & $1.3582414 \ldots$ & ] $1.35795,1.35805[$ \\
0.6 & $1.375657 \ldots$ & ] $1.37522545,1.3752256[$ \\
0.5 & $1.39533671 \ldots$ & ] $1.3946748,1.3946749[$ \\
0.4 & $1.4177921 \ldots$ & ] $1.4168798,1.4168801[$ \\
0.3 & $1.4437166 \ldots$ & ] $1.442575,1.4425755[$ \\
0.2 & $1.47402169 \ldots$ & ] $1.472798,1.472802[$ \\
0.1 & $1.5100616 \ldots$ & ] $1.509082,1.509084[$ \\
0 & $1.55377397 \ldots$ & ] $1.55377393,1.553774[$ \\
\hline \hline
\end{tabular}

$\left(J_{4}=J\right.$ and $\left.J_{4}=0\right)$, Wosiek's criterion gives the correct answer, but otherwise it always gives smaller values than the exact ones, although it fails by only a small margin $(\leqslant 0.1 \%)$.

\section{TWO-LAYER SQUARE LATTICE ISING MODEL}

Wosiek $^{1}$ predicted from his criterion that the critical temperature for the two-layer Ising model in the limit $L \rightarrow \infty$ should be $\beta_{c}=0.2656 \ldots$. This prediction has recently been shown to be incorrect, and better results obtained from higher-order moments of the transfer matrix were reported, ${ }^{3,4}$ as well as results from Monte Carlo simulations. ${ }^{4}$ We have also performed Monte Carlo simulations of this system, confirming the latter results. We used several lattice sizes, ranging from $L=5$ to $L=30$, and found that the pseudocritical temperature (temperature for which the specific heat is maximum) decreased with increasing $L$, starting from a value smaller than Wosiek's prediction, thus ruling out that prediction. Our sampling was not sufficiently accurate to test the precise values given in Refs. 3 and 4, but finite-size scaling analysis yielded a rough estimate for $\beta_{c}(\infty)$ which is compatible with those values.

\section{CONCLUSIONS}

We conclude from our tests that Wosiek's criterion, at least in its simplest form, given by the second moment of $T$, is not exact except for some special, highly symmetrical cases. In Secs. II, III, and IV we showed, by means of examples, that the existence of symmetry relations, such as self-duality, which determine the critical point, is not a sufficient condition for its validity, and in Sec. II we pointed out that the method is sensitive to the choice of the transfer matrix, and that the symmetry with respect to the anisotropic coupling constants is not preserved for all the possible choices. We attempted to reformulate the method to recover this later symmetry. The failure to reproduce the correct critical temperature for the two-layer Ising model, which is not self-dual and does not have any other symmetries which determine its critical point, suggests that the existence of such symmetries is a necessary condition for the validity of the maximum principle. ${ }^{3,4}$ As it stands, it still remains to be understood what class of models comply to the maximum principle, what is the mechanism underlying its validity (or not) in each case, and whether there is any generalization with a wider applicability. One possibility, explored in Refs. 3 and 4 , is that Wosiek's criterion is the lowest-order approximation in a hierarchy which converges to the exact result. It would be interesting to further explore this possibility by using higher moments of the transfer matrix to study symmetrical (e.g., self-dual) but anisotropic models, such as the anisotropic Ising model, taking into consideration the discussion in Sec. II.

\section{ACKNOWLEDGMENTS}

We are indebted to Professor José Luis Martins for his encouragement and for a critical reading of the manuscript. This work was supported by PRAXIS XXI Grants Nos. BD5037/95 and BD5704/95.
*Present address: Department of Physics, University of Illinois at Urbana-Champaign, Urbana, IL 61801-3080.

${ }^{1}$ J. Wosiek, Phys. Rev. B 49, 15023 (1994).

${ }^{2}$ R. J. Baxter, in Exactly Soluble Models in Statistical Mechanics
(Academic, London, 1984).

${ }^{3}$ Alessandro Pelizzola, Phys. Rev. B 51, 12005 (1995).

${ }^{4}$ P. Bialas, Z. Burda, P. Sawicki, and J. Wosiek, Phys. Rev. B 51, 12007 (1995). 